\title{
Genetic linkage and imprinting effects on body mass index in children and young adults
}

\author{
Olga Y Gorlova ${ }^{1}$, Christopher I Amos ${ }^{*, 1}$, Nancy W Wang ${ }^{1}$, Sanjay Shete ${ }^{1}$, Stephen T Turner ${ }^{2}$ \\ and Eric Boerwinkle ${ }^{3}$
}

${ }^{1}$ Department of Epidemiology, The University of Texas MD Anderson Cancer Center, Houston, TX, USA; ${ }^{2}$ Division of Hypertension, Department of Internal Medicine, Mayo Clinic, Rochester, MN, USA; ${ }^{3}$ Human Genetics Center and Institute of Molecular Medicine, The University of Texas Health Science Center, Houston, TX, USA

Body mass index (BMI) is used as a measure of fatness. Here we performed a genome-wide scan for genes related to BMI, while allowing for the possible effects of imprinting. We applied a sib pair linkage analysis to a sample of primarily children and young adults by using the Haseman-Elston method, which we modified to model the separate effects of paternally and maternally derived genetic factors. After stratification of sib pairs according to age, a number of regions showing linkage with BMI were identified. Most linkage and imprinting effects were found in children 5-11 years of age. Strongest evidences for linkage in children were found on chromosome 20 at 20p11.2-pter near the marker D20S851 $\left(L O D_{\text {Total }}=4.08, P=0.000046\right)$ and near the marker D20S482 $\left(L O D_{\text {Total }}=3.55, P=0.00016\right)$, and Chromosome 16 at 16p13 near the marker ATA41E04 $\left(L O D_{\text {Total }}=3.12, P=0.00025\right)$, and those loci did not show significant evidence for imprinting. Six regions showing evidence of imprinting were 3p23-p24 (paternal expression), 4q31.1-q32 (maternal expression), 10p14-q11 (paternal expression), and 12p12-pter (paternal expression) in children, and 4q31-qter (paternal expression) and 8p (paternal expression) in adults. European Journal of Human Genetics (2003) 11, 425-432. doi:10.1038/sj.ejhg.5200979

Keywords: BMl; linkage; imprinting; age grouping

\section{Introduction}

Numerous studies have shown that elevated Body mass index (BMI) is a risk factor for hypertension, heart diseases, diabetes, and cancers. ${ }^{1,2}$ Elevated BMI is also commonly used as a strong indicator of obesity. Since the metabolic pathways related to body mass are complex, ${ }^{3}$ one can expect several different loci to influence fatness and the risk for obesity. ${ }^{4}$ Indeed, a number of chromosomal regions linked to body mass-related traits have been suggested in several genome scans. ${ }^{5}$

Genomic imprinting is an epigenetic alteration of genes that leads to the expression or preferential expression of

*Correspondence: Dr Christopher I Amos, Department of Epidemiology, Box 189, The University of Texas MD Anderson Cancer Center, 1515 Holcombe Boulevard, Houston, TX 77030, USA. Tel: +1 713792 3020; Fax: +1 713792 0807; E-mail: camos@mdanderson.org

Received 23 September 2002; revised 13 January 2003; accepted 5 February 2003 an allele originating from only one parent. ${ }^{6}$ Comparative genomic studies as well as observations of some rare genetic syndromes in humans have identified many chromosomal regions that show imprinting (reviewed in Bartolomei and Tilghman ${ }^{7}$ ). Since imprinted genes are involved in many aspects of development, including prenatal and postnatal growth, ${ }^{7,8}$ imprinting may affect quantitative traits related to postnatal body size, ${ }^{9}$ and its effect could probably be better seen in children. To date, chromosomal regions such as $7 \mathrm{q}, 11 \mathrm{p} 15,15 \mathrm{q} 11-\mathrm{q} 13{ }^{7}$ and $20 \mathrm{q} 13,{ }^{10,11}$ harboring various genetic syndromes, have been shown to be imprinted. However, the complete extent of imprinting for the genome has not yet been determined.

One of the most popular hypotheses describing the evolution of imprinting is a sex-conflict theory. The theory states that conflict arises between the desire of the father to optimize his reproductive fitness by promoting the growth 
of the offspring even at the expense of the mother's future litters, and of the mother, who must conserve her own resources within any one litter to ensure that she does not have too many or too large offspring. ${ }^{7,12}$ The theory predicts that genes promoting prenatal and early postnatal growth are paternally expressed, whereas genes that suppress growth are maternally expressed.

Conventional linkage analyses of imprinted genes may fail to detect linkage, ${ }^{13,14}$ because parent-of-origin-dependent gene expression results in deviations from the laws of Mendelian genetics and an appropriate modeling of imprinted genes is needed. Recently, researchers started modeling potential effects of imprinting in the control of complex traits including obesity-related traits, both in adults $^{13,15}$ and newborns. ${ }^{16}$ However, in newborns one may have to control for strong maternal effects that affect intrauterine growth. Here we present the results of linkage analysis for BMI using 372 markers across the genome while allowing for the effects of imprinting. Our sample population includes participants with a range of ages from childhood through young adulthood, an age period not previously extensively studied in genetic linkage analyses of BMI. Yet, because of lesser cumulative environmental (dietary) exposure, the latter group, too, may be promising for genetic studies of body weight-related traits. We use age stratification, as we believe that different sets of genes affect body size at different ontogenetic stages. Genes involved in growth seem to be important in BMI control in children, which is probably not the case for adults. Age stratification may help reduce the heterogeneity and improve chances to detect linkage. Here we confirm several previously identified linkage regions and find several regions showing evidence of parent-of-origin effects.

\section{Materials and methods}

The sample that we studied comprised 1909 individuals who are members of 255 three-generation pedigrees who took part in the first phase of the Rochester Family Heart Study (RFHS). ${ }^{17}$ The RFHS is a population-based study initiated in 1984 to investigate the genetics of cardiovascular disease and its risk factors. Families participating in the study were ascertained on the basis of having at least two children enrolled in the schools of Rochester, Minnesota, and without regard to the health status of the family members. As many of their relatives as practicable were collected. The study population is over 99\% Caucasian. Height and weight measures were obtained by direct observation of subjects following well-established protocols. ${ }^{17}$ Subjects were asked to remove their shoes and outdoor clothing. Height was measured with a wall stadiometer and weight was determined on a beam balance.

Before the analysis, the extended families were divided into nuclear families to facilitate identity-by-descent calculations for the imprinting tests. ${ }^{14}$ Since different genes may affect BMI at different stages of ontogenesis, we not only analyzed the whole sample (893 sib pairs) but also the age-stratified groups. The groups were created to separate out effects that may exist during childhood, adolescence and early adulthood. We were also concerned that effects during adolescence might not be easily modeled given the potential heterogeneity of gene expression during this age period. The pubescent group was defined in this study as ages 12-16 inclusively and called 'adolescents'. Thus, the following sib-pair age groups were analyzed:

- children: both sibs were 5-11 years old (101 pairs from 69 independent sibships, 53 sibships having one sib pair, 237 subjects);

- adolescents: both sibs aged 12-16 years (91 pairs from 75 independent sibships, 67 sibships having one sib pair, 279 subjects).

- young adults, in which ages of both sibs were 17-30 years (173 pairs from 65 independent sibships, 41 sibships having one sib pair, 244 subjects).

The number of sib pairs in which both sibs were older than 30 was too small to perform a reliable analysis. Since some sib pairs included individuals from different age groups, the total number of sib pairs is greater than the sum of the sib pairs across the three age groups.

Before the linkage analysis, we adjusted BMI values for the effects of sex, age, and squared age (to account for possible nonlinearity of the dependency of BMI on age), separately for each age group, for standardization. The age groups and corresponding covariate adjustments are shown in Web Table 1. For children and adolescent age groups, the effects of adjustments were not significant though. We also explored the dependence of BMI on gender alone for the adolescent age group, having in mind that gender differences start to develop in that age group, but that was not significant either. When the sample of all sib pairs was analyzed, we tried two types of adjustment. One was based on the regression coefficients obtained for the whole sample, and the other was based on obtaining the residuals from regression analyses that were conducted within age strata. The results from either analysis were practically identical and so we only present the findings from the residuals after covariate adjustment within age strata.

DNA samples were evaluated using 372 minisatellite markers that are approximately evenly distributed along all the autosomes. Genotyping was performed by standard methods with an Applied Biosystems/Perkin-Elmer 377 automatic DNA sequencer. To minimize typing errors, all genotypes were performed in duplicate and any discrepancies were resolved by a senior laboratory supervisor. The proportions of alleles identical by descent with respect to each parent of origin were calculated by a modification of the IBD program from ACT package. ${ }^{14,18}$ For loci showing 
preliminary significant evidence for linkage and that were not in Hardy-Weinberg equilibrium, we applied a further modification of the IBD program to use genotype rather than allele frequencies for missing parents when calculating identity by descent. We applied Haseman-Elston tests $^{19}$ for linkage. The Haseman-Elston test evaluates the evidence in favor of linkage by regressing the squared pair differences for a trait onto the proportion of alleles identical by descent, $\pi$, for the marker locus. An alternative method to use for this study would have been the variance components method, but given the deviations from normality and kurtosis demonstrated by BMI (Web Table 2 ), this method can produce excess false-positive results. ${ }^{20}$ Thus, we choose the Haseman-Elston test over other variance-components procedures as more robust in our situation. To accommodate parent-of-origin effects, we used the extension of Haseman-Elston method as suggested by Hanson et al. ${ }^{13}$

We tested for the effects of parental-specific expressed loci by including two separate terms into the HasemanElston regression equation and considering two separate $\beta$ coefficients for the parental effects as follows:

$$
E\left[\left(x_{i}-x_{j}\right)^{2}\right]=\alpha+\beta_{M O} \pi_{M O i j}+\beta_{F A} \pi_{F A i j}
$$

where $x_{i}$ and $x_{j}$ are trait values for sibs, $\beta_{M O}$ and $\beta_{F A}$ are the genetic effects due to parental factors, and $\pi_{M O i j}$ and $\pi_{F A i j}$ are the estimated ibd sharing from mother and father (with range from 0 to 1 ), respectively.

To evaluate significance of total and parental-specific linkages, we used simulation. Unlinked marker data were generated using the SIMULATE program. ${ }^{21}$ This program assigns markers to individuals by sampling alleles for the founders in the pedigrees according to the specified allele frequencies, and assuming Hardy-Weinberg equilibrium. Then, markers are segregated to offspring. We generated families with exactly the same structure and the same BMI data, as we had observed for each age strata. Then the regression was performed using the observed trait values. We performed 40000 simulations for analysis of each $P$ value that is reported. The observed values of the total $t$ test for linkage as well as the parental-specific $t$-test values were used, separately, to derive total and parental-specific $P$-values. To obtain empirical $P$-values for the $t$-test values observed in our analysis, the $t$-test values were compared with the empirical distribution of $t$-values that were generated under the null hypotheses. The $P$-value was equal to the ratio of the number of simulated $t$-test values equal or exceeding the observed one, to the total number of tests performed.

We implemented a Bonferroni correction to paternaland maternal-specific $P$-values to allow for the two independent tests (maternally imprinted and paternally imprinted). This correction was not applied to the $P$-values for total linkage, as it is dependent on both maternal and paternal linkage. The $t$-test values that we obtained while performing linear regression of squared trait sib pair differences on identity by descent sharing were transformed into LOD scores by squaring the $t$-test values for those regressions that were negative and dividing by 4.6.

Significance of the test for imprinting (whether or not the two parental-specific regression coefficients are different) was also evaluated using a permutation procedure. Hanson et al. ${ }^{13}$ showed by simulation that type 1 error may be inflated for this test in presence of a nonimprinted locus linked to the marker when non-independent sib pairs were used. However, the type 1 error didn't exceed the nominal value if no trait locus was linked to the marker regardless of the number of sib pairs per family. This means that the distribution of the test $t$-statistic

$$
t=\frac{\beta_{F A}-\beta_{M O}}{\sqrt{\operatorname{Var} \beta_{F A}+\operatorname{Var} \beta_{M O}-2 \operatorname{Cov}\left(\beta_{F A}, \beta_{M O}\right)}}
$$

depends on whether or not there is a trait locus linked to the marker. ${ }^{13}$ In other words, the distribution is locusspecific. The permutation procedure was as follows: $\pi_{\mathrm{MO}}$ and $\pi_{\mathrm{FA}}$ were permuted within blocks defined by all sib pairs belonging to the same family, with probability 0.5 , independently for each family. Thus, we preserved the correlation in ibd within families while permuting the parental components and then derived an empirical distribution of the test for imprinting under the null hypothesis. The procedure was repeated 1000 times for each locus for which evidence for linkage and an indication to parent-of-origin effect was present. The $t$-test mentioned above was used as a test statistic and empirical critical values and $P$-values were derived separately for each locus.

Multipoint linkage analysis was used to help to choose between two following possibilities: effects from an imprinted trait locus versus effects from a locus that is not imprinted but is located at a different genetic distance from a marker in the two genders. The latter is possible when there is a large sex difference in recombination rates and can lead to the false conclusion that the locus is imprinted if single point analyses are conducted. ${ }^{13}$ Multipoint linkage analysis was done using Fulker-Cardon approxmation $^{22}$ for total linkage as well as for sex-specific linkages. As is shown in the Appendix, the approximation follows the same procedure with parent-of-origin-specific proportion of alleles shared IBD as with total IBD sharing. Sex-specific recombination distances were used. Each interval between markers was divided into such a number of intervals that the distance between points is approximately $1 \mathrm{cM}$ on the sex-average map, and the lengths of the intervals on the sex-specific maps were chosen accordingly. Thus, all the three maps were divided into the same number of intervals and the physical location of the corresponding points was close to being the same for both genders and the sex-average map. The results are presented graphically based on the sex-averaged scale. 
To check whether the markers were in Hardy-Weinberg equilibrium, we used the hwe program available from John Brzustowski (ftp://gause.biology.ualberta.ca/pub/jbrzusto/ cgi/hwe/), based on the method proposed in the paper by Guo and Thompson. ${ }^{23}$

\section{Results}

Of the 372 markers, 55 (14.8\%) were not in HardyWeinberg equilbrium with significance beyond the $5 \%$ level. Among the 21 markers that showed linkage, only three (about 14\%) showed departures from Hardy-Weinberg equilibrium, namely D8S1113, GATA49D12, and D20S851.

In the analysis of all sib pairs (without dividing them into age groups) we found a strong suggestive evidence for linkage near the marker D1S552 at $45.33 \mathrm{cM}$ from pter (1p36 region), thus confirming earlier findings it this region. ${ }^{24,25}$ The LOD score for total linkage equals 2.03 $\left(P_{\text {Lin }}=0.002\right)$. There was only a weakly suggestive evidence for imprinting for this locus, implying preferentially maternal expression $\left(L O D_{F A}=0.968, \quad P_{F A}=0.035\right.$; $\left.L O D_{M O}=1.538, P_{M O}=0.008\right)$.

Linkage and imprinting results after stratifying by age One might expect that the power of a linkage study would drop after a sample has been subdivided, because of the decrease in number of individuals. On the other hand, if genetically homogeneous subsamples can be isolated, evidence for linkage will increase. Here we present the results of analysis of age-stratified samples.

In the adolescent age group, no significant linkages were found. For both the children and the young adults there were several findings.

In children 5-11 years of age (Tables 1 and 2) there were 15 markers showing evidences for linkage to BMI, some of them strong with LOD scores greater than 4. Of those 15, two show only total linkage (one with LOD score higher than 3) and three more - both total and parent-specific linkage (two of them with $L O D_{\text {Total }}$ score higher than 3). A total of 10 markers show parent-specific linkage (i.e. linkage when taking parent-of-origin effect into account) only. Of them, we observed seven markers that showed only suggestive paternal expression (chromosomes 3, 10, $12,16,18)$. Three markers showed only suggestive maternal expression (chromosomes 2 and 4).

Chromosomes 2, 16, 18, and 20 show more than one marker linked to BMI. Two loci on chromosome 16, D16S404 and D16S764, 12 cM apart from each other, possibly reflect a presence of one major gene in the corresponding region. They show total and paternal linkage, the LOD score being higher for total linkage for both the markers. The strongest linkage was found on chromosome 20 with marker D20S851. The marker shows both total and maternal linkage, and again, the LOD score for the total linkage is higher than for maternal linkage only. Another marker from chromosome 20 and a marker from chromosome 5 show significant total linkage only.

As nominal $P$-values for test for imprinting may be biased towards smaller values in case of total linkage, we used simulation-derived locus-specific $P$-values for imprinting tests (see Materials and methods). For children, we found four loci showing significant parent-of-origin effects. Three of them show paternal expression (D3S3038, D10S1423, and GATA49D12), and one shows maternal expression (D4S1629) (Table 2).

For young adults (ages 17-30), six markers showed linkage with BMI (Tables 3 and 4). The marker D4S2417 on chromosome 4 and the marker D8S277 on Chromosome 8 showed paternal expression, both demonstrating significance for the empirical test for imprinting. The markers D9S922 and D15S1007 (chromosomes 9 and 15, respec-

Table 1 Description of the markers showing linkage with BMI (ages 5-11 years)

\begin{tabular}{|c|c|c|c|c|c|}
\hline Marker name & $\begin{array}{l}\text { Cytological } \\
\text { localization }\end{array}$ & $\begin{array}{l}\text { Genetic map } \\
\text { localization }\end{array}$ & Sex with higher $\theta$ & Ratio of $\theta$ & $\begin{array}{c}\text { Percentage of sibpairs with both parents' } \\
\text { marker information missing }\end{array}$ \\
\hline D2S410 & $2 p 11.2-q 13$ & 125.18 & Females & 1.9 & 26.7 \\
\hline D2S442 & $2 \mathrm{p} 14-\mathrm{q} 11.2$ & 147.4 & Females & 1.25 & 24.8 \\
\hline D3S3038 & $3 p 23-p 24$ & 44.81 & Females & 10.1 & 25.7 \\
\hline D4S1629 & $4 q^{3} 1.1-q_{33}$ & 157.99 & Females & 5 & 28.6 \\
\hline D5S1471 & $5 q 34-q 35$ & 172.13 & _ * & - & 34.3 \\
\hline D10S1423 & 10p14-q11.2 & 46.23 & Females & 6.75 & 36.2 \\
\hline GATA49D12 & 12p12-pter & 17.72 & Females & 1.44 & 27.6 \\
\hline ATA41E04 & $16 p 13$ & 11.46 & Males & 2.2 & 28.6 \\
\hline D16S404 & $16 \mathrm{p} 13$ & 18.07 & Males & 1.31 & 38.1 \\
\hline D16S764 & 16p11.2-p13.1 & 29.97 & No diff & 1 & 32.4 \\
\hline D16S3103 & 16p11.2-p13.1 & 32.07 & Females & 1.38 & 35.2 \\
\hline D18S843 & 18p11.2-p11.32 & 28.1 & Females & 1.71 & 39.0 \\
\hline D18S478 & $18 q 11.2-12.2$ & 52.86 & Females & 9.75 & 26.7 \\
\hline D20S482 & 20p12-p13 & 12.12 & - & - & 24.8 \\
\hline D20S851 & 20p11.2-pter & 24.7 & Females & 1.73 & 32.4 \\
\hline
\end{tabular}

*Sex-specific data on recombination are only provided for loci showing at least suggestive imprinting. 
Table 2 Characteristics of linkage found in age group 5-11 years

\begin{tabular}{|c|c|c|c|c|c|c|c|}
\hline \multirow[b]{2}{*}{ Marker name } & \multicolumn{3}{|c|}{ LOD scores } & \multicolumn{3}{|c|}{ P-value } & \multirow{2}{*}{$\begin{array}{l}\text { Test for imprinting } \\
\text { (empirical P-value) }\end{array}$} \\
\hline & Total & Paternal & Maternal & Total & Paternal & Maternal & \\
\hline D2S410 & 1.458 & 0.089 & 1.77 & 0.0059 & 0.528 & 0.006 & 0.3 \\
\hline D2S442 & 0.785 & 0.000 & 2.4 & 0.0316 & 1.0 & 0.00146 & 0.27 \\
\hline D3S3038 & 0.231 & 1.77 & 0.000 & 0.1511 & 0.0065 & 1.0 & 0.036 \\
\hline D4S1629 & 0.226 & 0.000 & 1.89 & 0.1537 & 1.0 & 0.005 & 0.025 \\
\hline D5S1471 & 2.48 & 0.651 & 1.717 & 0.0006 & 0.098 & 0.007 & - \\
\hline D10S1423 & 0.308 & 1.89 & 0.000 & 0.1183 & 0.005 & 1.0 & 0.04 \\
\hline GATA49D12 & 0.536 & 1.83 & 0.000 & 0.0609 & 0.006 & 1.0 & 0.027 \\
\hline ATA41E04 & 0.385 & 1.82 & 0.000 & 0.0952 & 0.006 & 1.0 & 0.34 \\
\hline D16S404 & 3.12 & 2.03 & 0.931 & 0.00025 & 0.004 & 0.046 & 0.5 \\
\hline D16S764 & 2.45 & 2.14 & 0.379 & 0.0006 & 0.003 & 0.195 & 0.58 \\
\hline D16S3103 & 1.284 & 2.2 & 0.010 & 0.0095 & 0.003 & 0.830 & 0.36 \\
\hline D18S843 & 1.110 & 2.1 & 0.000 & 0.0142 & 0.004 & 1.0 & 0.26 \\
\hline D18S478 & 0.509 & 2.31 & 0.000 & 0.0668 & 0.002 & 1.0 & 0.075 \\
\hline D20S482 & 3.55 & 1.348 & 1.608 & 0.00016 & 0.017 & 0.009 & - \\
\hline D20S851 & 4.08 & 0.420 & 3.39 & 0.000046 & 0.181 & 0.00019 & 0.175 \\
\hline
\end{tabular}

${ }^{a} P$-values were empirically derived. Parental-specific $P$-values are Bonferroni corrected.

${ }^{\mathrm{b}}$ Test for imprinting shows if there is a significant difference between regression coefficients for maternal IBD sharing vs paternal IBD sharing ${ }^{12}$; $P$-values were obtained by permutation.

Table 3 Description of the markers showing linkage with BMI (ages 17-30 years)

\begin{tabular}{|c|c|c|c|c|c|}
\hline $\begin{array}{l}\text { Name of the } \\
\text { marker }\end{array}$ & $\begin{array}{l}\text { Cytological } \\
\text { localization }\end{array}$ & $\begin{array}{c}\text { Localization } \\
\text { on genetic map }\end{array}$ & $\begin{array}{l}\text { Sex with } \\
\text { higher } \theta\end{array}$ & $\begin{array}{c}\text { Ratio of higher } \theta \\
\text { to lower one }\end{array}$ & $\begin{array}{l}\text { Percentage of sibpairs } \\
\text { with both parents' marker } \\
\text { information missing }\end{array}$ \\
\hline D4S2417 & Distal from 4q31 & 181.93 & Males & 1.4 & 36.4 \\
\hline D8S277 & $8 p$ & 8.34 & Females & 1.2 & 37.5 \\
\hline D8S1113 & $8 p 11-q 13$ & 77.89 & - & - & 45.1 \\
\hline D9S922 & $9 \mathrm{q} 21-\mathrm{q} 22.1$ & 80.31 & Females & 1.5 & 39.3 \\
\hline D14S742 & $14 q^{11} .2-21.3$ & 12.46 & - & - & 34.1 \\
\hline D15S1007 & $15 q 12-q 21.1$ & 25.86 & None & 1 & 39.3 \\
\hline
\end{tabular}

Table 4 Characteristics of linkage found in the age group 17-30 years

\begin{tabular}{|c|c|c|c|c|c|c|c|}
\hline \multirow[b]{2}{*}{ Name of the marker } & \multicolumn{3}{|c|}{ LOD scores } & \multicolumn{3}{|c|}{ P-value $e^{a}$} & \multirow{2}{*}{$\begin{array}{l}\text { Test for imprinting } \\
\text { (empirical P-value }\end{array}$} \\
\hline & Total & Paternal & Maternal & Total & Paternal & Maternal & \\
\hline D4S2417 & 0.379 & 1.84 & 0 & 0.095 & 0.005 & 1.0 & 0.0095 \\
\hline D8S277 & 0.801 & 1.98 & 0 & 0.029 & 0.003 & 1.0 & 0.013 \\
\hline D8S1113 & 2.05 & 1.87 & 0.0026 & 0.00135 & 0.0045 & 0.91 & 0.072 \\
\hline D9S922 & 1.249 & 0.11 & 1.85 & 0.008 & 0.485 & 0.004 & 0.18 \\
\hline D14S742 & 1.95 & 0.496 & 1.717 & 0.002 & 0.137 & 0.006 & - \\
\hline D15S1007 & 0.704 & 0 & 1.87 & 0.037 & 1.0 & 0.004 & 0.48 \\
\hline
\end{tabular}

a Parental-specific P-values are Bonferroni corrected.

tively) showed suggestive maternal expression, although the test for imprinting was not significant. The marker D14S742 on chromosome 14 demonstrated suggestive total linkage without effects of imprinting. The marker D8S1113 on chromosome 8 showed both total linkage and suggestive paternal expression, although the test for imprinting was not significant.
There were no markers that showed significant linkage to BMI simultaneously in both young adults and children. However, markers in the chromosomal region 4q31-q33 showed linkage in both the groups, but over a $24 \mathrm{~cm}$ region. Also, the imprinting effects were different in this region, with children showing maternal expression and young adults showing paternal expression. 
The results for all chromosomes are presented in Web Figure 2, which shows the findings for each locus on each chromosome for the children and for the young adults.

\section{Discussion}

Published genome scans and other studies of BMI have identified numerous chromosomal regions showing linkage or association (reviewed in Rankinen et $a l^{5}$ ). Different studies tend to identify different regions. These 'inconsistencies' might, in part, result from the use of samples of different ethnic origins and with different disease statuses. The examples of such a diversity include analyses of Mexican-Americans; ${ }^{26}$ Pima Indians predisposed to type II diabetes; ${ }^{13,27}$ Finnish diabetic II families ascertained through an affected sib pair; ${ }^{28}$ Finnish families having obese subjects; ${ }^{29}$ Ashkenazi Jewish families ascertained for type II diabetes; ${ }^{30}$ Amish; ${ }^{31}$ Dutch dyslipidemic families; ${ }^{32}$ see Rankinen et $a l^{5}$ for the complete list of the linkage studies. Different populations may be polymorphic for different loci involved in control of BMI. In addition, adjustment for different covariates also can modify results. $^{32}$

In this study, we found evidence for linkage with 22 markers that are located in 17 distinct regions. Of those, 11 regions, namely 1p36, 4q31-q32, 8p11-p12, 10p, 12p12p13, 14p11.2, 15q11-13, 16p, 18p11.2, 18q12, 20p12 have been reported previously (see Obesity map update ${ }^{5}$ ) from linkage or association studies. Regions 15q11-q13 and 20 p12 are known to harbor obesity-related Mendelian disorders (Angelman syndrome with obesity/Prader-Willi syndrome and Bardet-Biedl syndrome 6 , respectively). The 3 p24.1 region that appeared in our analysis was identified recently as suggestively linked to BMI in a combined analysis of genome scans for obesity. ${ }^{33}$ Several other regions found in the present study were previously shown to be associated or linked with other obesity-related phenotypes: 2 p12 is associated with triceps skinfold, $9 q 22.1$ is linked to abdominal subcutaneous fat, $18 \mathrm{q} 12$ is linked to fat-free mass. ${ }^{5}$ The only region found here that has not been reported previously is $5 \mathrm{q} 34-\mathrm{q} 35$ that shows total linkage (no parent-of-origin effect).

We found several regions showing parent-of-origin effect. Most of them, namely 3p23-24, 4q31-q33, 8p, 12p12-pter (Tables 2 and 4) have not been previously reported as imprinted. Interestingly, for all those regions there were conventional linkage findings with LODs from 1.8 to 3.2 (see Table 4 in Rankinen et $a l^{5}$ ).

Hanson et $a l^{13}$ and Lindsay et al ${ }^{15}$ found a tentative evidence for imprinting in the region $10 \mathrm{p}$, however, their linkage (at $20 \mathrm{~cm}$ ) is $26 \mathrm{~cm}$ apart from the peak observed in the present study (at $46 \mathrm{~cm}$ ). We were unable to identify a peak that Lindsay et $a l^{15}$ found on chromosome $5(71 \mathrm{cM}$ from pter) for maternal expression. In the study of BMI in newborns by that group, ${ }^{16}$ a peak on Chromosome 11 at
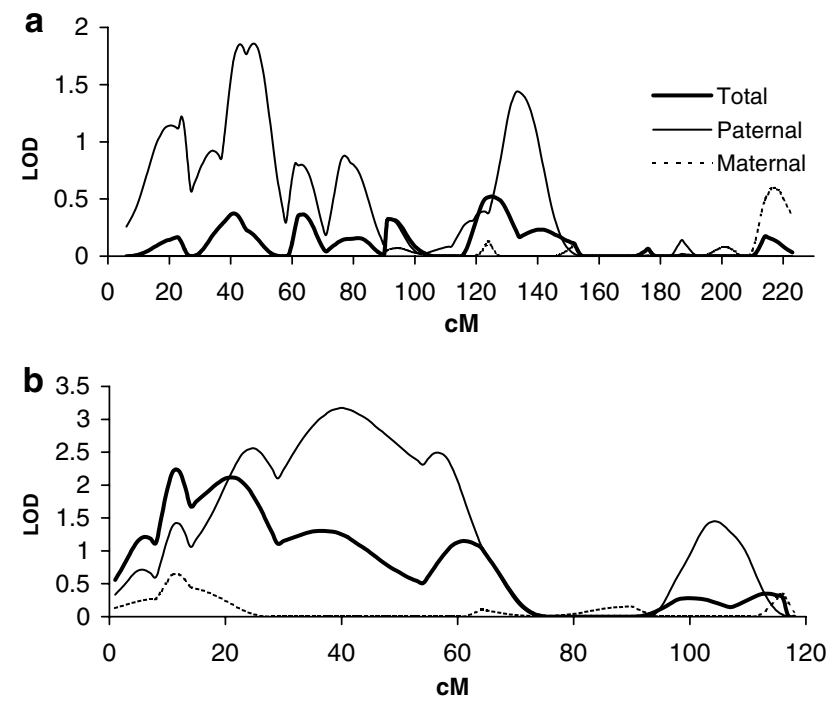

Figure 1 Results of multipoint linkage analysis (total and sex-specific linkages) for BMI in children 5-11 years old. (a) Chromosome 3; (b) Chromosome 18.

$85 \mathrm{~cm}$ (paternal expression) was found. We observed modest peaks on Chromosome 11 in children - one at $85 \mathrm{~cm}$, LOD 0.85, another at $100 \mathrm{~cm}$ from pter, $\mathrm{LOD}=1.6$, both showing paternal expression. In fact, the location of the former one, as well as the mode of imprinting, coincides with that of the peak reported by Lindsay et al ${ }^{15}$ Interestingly, other previously known imprinted regions on chromosomes $7 \mathrm{q} 22-\mathrm{q} 31$ and $15 \mathrm{q} 11-\mathrm{q} 13^{8}$ produce clear, although in most cases modest, peaks and show only parent-specific linkage in our analysis (Figure 1). In adults, the peak at 15q12-22 reaches a borderline significance $(P=0.004)$ for maternal expression, although the test for imprinting is not significant. A comparison of the results of our study and a list of genes that may influence body mass ${ }^{5}$ allowed us to suggest candidate genes in many regions identified in our study (Web Table 3).

Our study emphasizes the importance of adequate sample grouping. This can be illustrated by the fact that in the analysis of all possible sib pairs we found only one region, 1p36, with suggestive linkage even though many more pairs were analyzed than in the other (stratified) analyses. Many more linkages were found after sample stratifying. The explanation may be as follows. There are different sets of genes responsible for body size in children and in adults. As BMI depends on both weight and height, in children it will be controlled in part by genes involved in growth. Owing to the fact that many imprinted genes participate in early growth control, one can expect that effects of imprinting in children should be stronger, which we indeed observed.

Finally, we would like to discuss the problem of falsepositive results. In our study we used a threshold for LOD 
scores of 1.75, which corresponds to one false positive per genome scan with 400 markers. ${ }^{34}$ The LOD scores that we are reporting reflect three different tests. However, the test for total linkage is completely dependent on the two parent-specific tests. Therefore, when we applied a Bonferroni correction we adjusted for two independent tests. We detected no linkage in the adolescent group, which had approximately the same number of sib pairs as the group of children. This conforms to our expectations of greater genetic heterogeneity in this age range and may serve as an indirect argument that (some of) the linkages found in children and in adults are real. By applying permutation to generate empirical $P$-values, we addressed the issue of possible type 1 error inflation for the test for imprinting when there is a nonimprinted trait locus linked to a marker. We assessed significance of linkage findings using a permutation approach that assumes the markers are in Hardy-Weinberg equilibrium. Only one marker, D8S1113, that was in Hardy-Weinberg disequilbrium showed a significant excess of homozygotes $\left(\chi^{2}=38.24\right.$, $P<0.00001)$. Marker GATA49D12 showed an insignificant excess of heterozygotes, while marker D20S851 showed an insignificant excess of homozygotes. The excess frequency of homozygotes for D8S1113 would decrease the empirical power to detect linkage compared with the simulated power used to compute $P$-values. For the other two markers, departure from Hardy-Weinberg equilibrium (owing to variation in heterozygous genotypes from expected frequencies) would not affect the power to detect linkage and so should not affect the simulated $P$-values.

Sex differences in recombination frequency, in the presence of a nonimprinted major gene in the region, can lead to artefactual detection of parent-of-origin effects, although the sex difference must be very high (at least 10fold). ${ }^{13}$ A weaker linkage in the sex with higher recombination in that region could be erroneously interpreted as imprinting. Such a phenomenon might explain our singlemarker results for the markers D3S3038 and D18S478 (Table 2). To minimize this confounding, we used a modified Fulker-Cardon approach for multipoint linkage analysis with sex-specific recombination rates on Chromosomes 3 and 18. If there were a trait locus that is not imprinted and genetically located in females much farther from a marker than in males, we would see an increase in female linkage as the locus is approached. However, this is not the case (Figure 1). We thus conclude that the putative loci located near the markers D3S3038 and D18S478 are imprinted. However, a much denser set of markers would be desirable to evaluate imprinting versus sex-specific recombination. The wide peak found on Chromosome 18 at $29-54 \mathrm{~cm}$ is absent in the single-marker analysis. The difference is caused by the absence of the marker D18S542 in the multipoint analysis. The marker was not used because its location on the sex-specific maps remains unspecified.
In conclusion, several of the loci we identified in children showed strong evidence for linkage and their further investigation in homogeneously young datasets would be of interest.

\section{Acknowledgements}

The study was supported by Grants HL51021, ES09912, and HG02275.

\section{References}

1 Field AE, Coakley EH, Must A et al: Impact of overweight on the risk of developing common chronic diseases during a 10-year period. Arch Intern Med 2001; 161: 1581-1586.

2 Horwich TB, Fonarow GC, Hamilton MA, MacLellan WR, Woo MA, Tillisch JH: The relationship between obesity and mortality in patients with heart failure. J Am Coll Cardiol 2001; 38: 789-795.

3 Froguel P, Boutin P: Genetics of pathways regulating body weight in the development of obesity in humans. Exp Biol Med 2001; 226: 991-996.

4 Comuzzie AG, Williams JT, Martin LJ, Blangero J: Searching for genes underlying normal variation in human adiposity. J Mol Med 2001; 79: 57-70.

5 Rankinen T, Perusse L, Weisnagel SJ, Snyder EE, Chagnon YC, Bouchard C: The human obesity gene map: the 2001 update. Obes Res 2002; 10: 196-243.

6 Tilghman SM: The sins of the fathers and mothers: genomic imprinting in mammalian development. Cell 1999; 96: 185-193.

7 Bartolomei MS, Tilghman SM: Genomic imprinting in mammals. Annu Rev Genet 1997; 31: 493-525.

8 Falls JG, Pulford DJ, Wylie AA, Jirtle RL: Genomic imprinting: implications for human disease. Am J Pathol 1999; 154: 635-647.

9 Le Stunff C, Fallin D, Bougneres P: Paternal transmission of the very common class I INS VNTR alleles predisposes to childhood obesity. Nat Genet 2001; 29: 96-99.

10 Hayward BE, Moran V, Strain L, Bonthron DT: Bidirectional imprinting of a single gene: GNAS1 encodes maternally, paternally, and biallelically derived proteins. Proc Natl Acad Sci USA 1998; 22: 15475-15480.

11 Shore EM, Ahn J, Jan de Beur S et al: Paternally inherited inactivating mutations of the GNAS1 gene in progressive osseous heteroplasia. N Engl J Med 2002; 346: 99-106.

12 Moore T, Haig D: Genomic imprinting in mammalian development: a parental tug-of-war. Trends Genet 1991; 7: 45-49.

13 Hanson RL, Kobes S, Lindsay RS, Knowler WC: Assessment of parent-of-origin effects in linkage analysis of quantitative traits. Am J Hum Genet 2001; 68: 951-962.

14 Shete S, Amos CI: Testing for genetic linkage in families by variance components approach in the presence of genomic imprinting. Am J Hum Genet. 2002; 70: 751-757.

15 Lindsay RS, Kobes S, Knowler WC, Bennett PH, Hanson RL: Genome-wide linkage analysis assessing parent-of-origin effects in the inheritance of type 2 diabetes and BMI in Pima Indians. Diabetes 2001; 50: 2850-2857.

16 Lindsay RS, Kobes S, Knowler WC, Hanson RL: Genome-wide linkage analysis assessing parent-of-origin effects in the inheritance of birth weight. Hum Genet 2002; 110: 503-509.

17 Turner ST, Weidman WH, Michels VV et al: Distribution of sodium-lithium countertransport and blood pressure in Caucasians five to eighty-nine years of age. Hypertension 1989; 13: 378-391.

18 De Andrade M, Krushkal J, Yu L, Zhu D, Amos CI: ACT - A computer package for analysis of complex traits. Am J Hum Genet 1998; 63: A287 (abstract).

19 Haseman JK, Elston RC: The investigation of linkage between a quantitative trait and a marker locus. Behav Genet 1972; 2: 3-19. 
20 Allison DB, Neale MC, Zannolli R, Schork NJ, Amos CI, Blangero $\mathrm{J}$ : Testing the robustness of the likelihood-ratio test in a variancecomponent quantitative-trait loci-mapping procedure. Am J Hum Genet 1999; 65: 531-544.

21 Terwilliger J, Ott J: Handbook of human genetic linkage. Baltimore: Johns Hopkins University Press, 1994.

22 Fulker DW, Cardon LR: A sib-pair approach to interval mapping of quantitative trait loci. Am J Hum Genet 1994; 54: 1092-1103.

23 Guo SW, Thompson EA: Performing the exact test of HardyWeinberg proportion for multiple alleles. Biometrics 1992; 48: 361-372.

24 Wilson AF, Elston RC, Tran LD, Siervogel RM: Use of the robust sib-pair method to screen for single-locus, multiple-locus, and pleiotropic effects: application to traits related to hypertension. Am J Hum Genet 1991; 48: 862-872.

25 Fernandez-Real JM, Vendrell J, Ricart W et al: Polymorphism of the tumor necrosis factor-alpha receptor 2 gene is associated with obesity, leptin levels, and insulin resistance in young subjects and diet-treated type 2 diabetic patients. Diabetes Care 2000; 23 : 831-837.

26 Mitchell BD, Cole SA, Comuzzie AG et al: A quantitative trait locus influencing BMI maps to the region of the beta-3 adrenergic receptor. Diabetes 1999; 48: 1863-1867.

27 Hanson RL, Ehm MG, Pettitt DJ et al: An autosomal genomic scan for loci linked to type II diabetes mellitus and body-mass index in Pima Indians. Am J Hum Genet 1998; 63: 1130-1138.

28 Watanabe RM, Ghosh S, Langefeld CD et al: The Finland-United States investigation of non-insulin-dependent diabetes mellitus genetics (FUSION) study. II. An autosomal genome scan for diabetes-related quantitative-trait loci. Am J Hum Genet 2000; 67: 1186-1200.

29 Ohman M, Oksanen L, Kaprio J et al: Genome-wide scan of obesity in Finnish sibpairs reveals linkage to chromosome Xq24. J Clin Endocrinol Metab 2000; 85: 3183-3190.

30 Permutt MA, Wasson JC, Suarez BK et al: A genome scan for type 2 diabetes susceptibility loci in a genetically isolated population. Diabetes 2001; 50: 681-685.

31 Hsueh WC, Mitchell BD, Schneider JL et al: Genome-wide scan of obesity in the Old Order Amish. J Clin Endocrinol Metab 2001; 86: 199-1205.

32 Van der Kallen CJ, Cantor RM, van Greevenbroek MM et al: Genome scan for adiposity in Dutch dyslipidemic families reveals novel quantitative trait loci for leptin, body mass index and soluble tumor necrosis factor receptor superfamily 1A. Int J Obes Relat Metab Disord 2000; 24: 1381-1391.

$33 \mathrm{Wu} \mathrm{X}$, Cooper RS, Borecki I et al: A combined analysis of genomewide linkage scans for body mass index from the National Heart, Lung, and Blood Institute Family Blood Pressure Program. Am J Hum Genet 2002; 70: 1247-1256.

34 Rao DC, Gu C: False positives and false negatives in genome scans. Adv Genet 2001; 42: 487-498.

\section{Appendix A}

Interval mapping approach for multipoint parent-specific ibd computation.

Let $\pi_{1 f}$ and $\pi_{2 f}$ be proportion of paternally derived alleles shared ibd by the sibs at two consecutive markers $M_{1}$ and
$M_{2}$. Let $\theta_{m, 12}$ be sex-specific (male) recombination fraction between these two flanking markers. Let $M_{q}$ be a site in between these two flanking markers and let and $\theta_{m, 1}$ and $\theta_{m, 2}$ be recombination fractions, based on male map, $M_{1}$ and $M_{q}$ and $M_{2}$ and $M_{q}$, respectively. We use and $\pi_{1 f}$ and $\pi_{2 f}$ to obtain $\pi_{q f}$, the proportion of paternally derived alleles shared ibd by the sibs at the site $M_{q}$. We use the interval mapping approach proposed by Fulker and Cardon ${ }^{22}$ by using a linear regression equation with $\pi_{1 f}$ and $\pi_{2 f}$ as predictors.

The regression equation we use is

$$
\pi_{q f}=\alpha+\beta_{\pi_{1 f}} \pi_{1 f}+\beta_{\pi_{2 f}} \pi_{2 f}
$$

Then, estimators of $\beta_{\pi_{1 f}}, \beta_{\pi_{2 f}}$, and $\alpha$ are given by a

$$
\begin{gathered}
\hat{\beta}_{\pi_{1 f}}=\frac{\operatorname{Var}\left(\pi_{2 f}\right) \operatorname{Cov}\left(\pi_{1 f}, \pi_{q f}\right)-\operatorname{Cov}\left(\pi_{1 f}, \pi_{2 f}\right) \operatorname{Cov}\left(\pi_{2 f}, \pi_{q f}\right)}{\operatorname{Var}\left(\pi_{1 f}\right) \operatorname{Var}\left(\pi_{2 f}\right)-\left(\operatorname{Cov}\left(\pi_{1 f}, \pi_{2 f}\right)\right)^{2}} \\
\hat{\beta}_{\pi_{2 f}}=\frac{\operatorname{Var}\left(\pi_{1 f}\right) \operatorname{Cov}\left(\pi_{2 f}, \pi_{q f}\right)-\operatorname{Cov}\left(\pi_{1 f}, \pi_{2 f}\right) \operatorname{Cov}\left(\pi_{2 f}, \pi_{q f}\right)}{\operatorname{Var}\left(\pi_{1 f}\right) \operatorname{Var}\left(\pi_{2 f}\right)-\left(\operatorname{Cov}\left(\pi_{1 f}, \pi_{2 f}\right)\right)^{2}}
\end{gathered}
$$

and

$$
\hat{\alpha}=E\left(\pi_{q f}\right)-\hat{\beta} \pi_{1 f} E\left(\pi_{1 f}\right) \hat{\beta} \pi_{2 f} E\left(\pi_{2 f}\right)
$$

From Table 2 of Shete and Amos, ${ }^{14}$ we obtain $E\left(\pi_{f}\right)=1 / 2$, $E\left(\pi_{f}\right)=1 / 4$, and $\operatorname{Cov}\left(\pi_{1 f}, \pi_{2 f}\right)=\left(1-2 \theta_{m}\right)^{2} / 4$. Using these values in above equations, we obtain estimates of $\beta$ 's and $\alpha$ which are given below:

$$
\begin{gathered}
\hat{\beta}_{\pi 1 f}=\frac{\left(1-2 \theta_{m, 1}\right)^{2}-\left(1-2 \theta_{m, 12}\right)^{2}\left(1-2 \theta_{m, 2}\right)^{2}}{1-\left(1-2 \theta_{m, 12}\right)^{2}} \\
\hat{\beta}_{\pi 2 f}=\frac{\left(1-2 \theta_{m, 2}\right)^{2}-\left(1-2 \theta_{m, 12}\right)^{2}\left(1-2 \theta_{m, 1}\right)^{2}}{1-\left(1-2 \theta_{m, 12}\right)^{2}}
\end{gathered}
$$

and

$$
\hat{\alpha}=\frac{1}{2}\left(1-\hat{\beta}_{\pi_{1 f}}-\hat{\beta}_{\pi_{2 f}}\right)
$$

For any given site between the two markers $M_{1}$ and $M_{2}$, one can convert the distances into recombination fractions using one of the available mapping functions and then obtain the parent-specific multipoint ibd sharing by using equation (A.1). The proportion of maternally derived alleles shared ibd by sibs is similarly obtained. 